\title{
GLOBALS OF PSEUDOVARIETIES OF COMMUTATIVE SEMIGROUPS: THE FINITE BASIS PROBLEM, DECIDABILITY AND GAPS
}

\author{
JORGE ALMEIDA $^{1}$ AND ASSIS AZEVEDO ${ }^{2}$ \\ ${ }^{1}$ Centro de Matemática, Faculdade de Ciências, Universidade do Porto, \\ 4099-002 Porto, Portugal \\ ${ }^{2}$ Centro de Matemática, Universidade do Minho, Braga, Portugal
}

(Received 4 May 1999)

\begin{abstract}
Whereas pseudovarieties of commutative semigroups are known to be finitely based, the globals of monoidal pseudovarieties of commutative semigroups are shown to be finitely based (or of finite vertex rank) if and only if the index is 0,1 or $\omega$. Nevertheless, on these pseudovarieties, the operation of taking the global preserves decidability. Furthermore, the gaps between many of these globals are shown to be big in the sense that they contain chains which are order isomorphic to the reals.
\end{abstract}

Keywords: semigroupoid; global pseudovariety; pseudoidentity basis

AMS 2000 Mathematics subject classification: Primary 20M07; 20M05

\section{Introduction}

Building on ideas of Rhodes and others $[\mathbf{1 5}, \mathbf{1 6}]$, Tilson $[\mathbf{1 7}]$ introduced categories and semigroupoids (categories without local identities) as tools for studying semidirect products of semigroups. Weil and Almeida [9] integrated the profinite perspective into Tilson's theory, culminating in the description of a basis of pseudoidentities for a semidirect product $\boldsymbol{V} * \boldsymbol{W}$ of pseudovarieties of semigroups depending on a basis of pseudoidentities for the global pseudovariety of semigroupoids $g \boldsymbol{V}$ generated by $\boldsymbol{V}$. The application of this basis to establish decidability of certain semidirect products has led Almeida to the notion of hyperdecidability [4], proving in particular that if $g \boldsymbol{V}$ is decidable and has vertex rank bounded by some given natural number and $\boldsymbol{W}$ is hyperdecidable, then $\boldsymbol{V} * \boldsymbol{W}$ is decidable. While the bounded vertex rank hypothesis has been relaxed by Almeida and Steinberg $[\mathbf{5}, \boldsymbol{6}]$ by slightly strengthening the other two hypotheses, the fact that many usual pseudovarieties are local (i.e. their globals have vertex rank 1), and those that are not have globals with small vertex rank (such as 2), prompted a deeper look into the vertex rank of globals. Moreover, the best-known cases of non-locality, namely those of the pseudovarieties $\boldsymbol{J}$ and $\mathbf{C o m}$ - consisting of all finite, respectively, $\mathcal{J}$-trivial and commutative semigroups $[\mathbf{1 3}, \mathbf{1 6}]$ (see also $[\mathbf{1 2}]$ ) - are both associated with a commutation 
phenomenon. While Rhodes has claimed that there are examples of pseudovarieties of semigroups whose globals have infinite vertex rank, no specific examples have hitherto been published.

In [10], Teixeira and the authors considered the finite basis problem for semidirect products of the forms $\boldsymbol{V} * \boldsymbol{D}$ and $\boldsymbol{V} * \boldsymbol{D}_{n}$ and its relationship with the finite basis problem for $\boldsymbol{V}$ and $g \boldsymbol{V}$. In particular, they showed that the problem may be systematically treated when $\boldsymbol{V}$ contains the five-element aperiodic Brandt semigroup $B_{2}$. So, the problem is only of interest for pseudovarieties excluding $B_{2}$, that is those consisting of semigroups in which regular $\mathcal{J}$-classes are subsemigroups.

In this paper, we deal with the case of pseudovarieties of commutative semigroups. Consider the profinite completion $\hat{\mathbb{N}}$ of the semiring $\mathbb{N}$ of non-negative integers and denote by $\omega$ its unique non-zero additive idempotent. Let $\mathbb{P}=\mathbb{N} \backslash\{0\}$ and $\hat{\mathbb{P}}=\hat{\mathbb{N}} \backslash\{0\}$. Each $\pi \in \hat{\mathbb{P}}$ may be viewed as a unary implicit operation $x \mapsto x^{\pi}$ on finite semigroups $[\mathbf{3}, \mathbf{8}]$. For $m \in \mathbb{P} \cup\{0, \omega\}$ and $\pi \in \hat{\mathbb{P}}$, let $\mathbf{C o m}_{m, \pi}$ denote the pseudovariety of all finite commutative semigroups satisfying the pseudoidentity $x^{m+\pi}=x^{m}$. Brzozowski and Simon [11] showed that the pseudovariety $\mathbf{C o m}_{1,1}$ is local. This result was extended by Almeida $[\mathbf{2}, \mathbf{3}]$ who showed that $\mathbf{C o m}_{1, k}$ is local for $k \in \mathbb{P} \cup\{\omega\}$. Thérien and Weiss [16] showed that the pseudovariety $\mathbf{C o m}=\mathbf{C o m}_{\omega, \omega}$ is not local and obtained a basis for the global $g \mathbf{C o m}$ consisting of a single pseudoidentity on a two-vertex graph. Straubing [15] (see also [3, 17]) showed that every non-trivial pseudovariety of groups is local, as a pseudovariety of monoids. As pseudovarieties of semigroups, pseudovarieties of groups are no longer local, the vertex rank of their globals is raised to $2[\mathbf{9}]$. Thus, the pseudovarieties of the form $g \mathbf{C o m}_{0, \pi}$ have vertex rank 2. We show that in fact $g \mathbf{C o m} m, \pi$ has finite vertex rank if and only if $m \in\{0,1, \omega\}$. We also show that $g \mathbf{C o m}_{m, \pi}$ is decidable if and only if $\mathbf{C o m}_{m, \pi}$ is decidable. It remains an open problem whether $g \boldsymbol{V}$ is decidable for every decidable pseudovariety $\boldsymbol{V}$ of semigroups.

The pseudovarieties of the form $\mathbf{C o m}_{m, \pi}$ are precisely the monoidal pseudovarieties of commutative semigroups, i.e. those that are generated by monoids. Taking $m \in \mathbb{P} \cup\{0\}$ and $\pi \in \mathbb{P}$, one obtains the pseudovariety analogue of Nelson's skeleton of varieties of commutative semigroups [14]. It follows from results of Almeida $[\mathbf{1}, \mathbf{3}]$ that between two consecutive skeleton points $\mathbf{C o m} m, k$ and $\mathbf{C o m}_{m^{\prime}, k^{\prime}}$ there are only countably many pseudovarieties and there are no infinite descending chains. In contrast, we show that, for $m, m^{\prime} \geqslant 2$, between their globals there is a chain of categorical pseudovarieties of semigroupoids (i.e. which are generated by their categories) which is isomorphic to the usual ordering of the real numbers.

Preliminary versions of this paper have been presented at seminars and conferences since 1996. The results have evolved considerably along the way and at present bear perhaps little resemblance to those presentations.

\section{Preliminaries}

For general background and undefined terms, the reader is referred to $[\mathbf{3 , 9 , 1 7}]$. In particular, a graph $\Gamma$ is a quadruple $\langle V, E, \alpha, \omega\rangle$, where $V$ is a set (of vertices), $E$ is a set (of edges) and $\alpha, \omega: E \rightarrow V$ are two functions. If $\langle V, E, \alpha, \omega\rangle$ is a graph and $a \in E$, then 
$\alpha(a)$ is the beginning of $a$ and $\omega(a)$ is the end of $a$. A loop in a graph is an edge whose ends coincide. By a path we mean a sequence of edges $a_{1} a_{2} \cdots a_{n}$ such that, for $k<n$, $\omega\left(a_{k}\right)=\alpha\left(a_{k+1}\right)$. If $u=a_{1} a_{2} \cdots a_{n}$ is a path, then the beginning and the end of the path, denoted by $\alpha(u)$ and $\omega(u)$, are $\alpha\left(a_{1}\right)$ and $\omega\left(a_{n}\right)$, respectively. For a path $u=a_{1} a_{2} \cdots a_{n}$ and an edge $a$ we denote by $|u|_{a}$ the number of indices $i \leqslant n$ such that $a_{i}=a$. The content of $u$ is defined as the set of edges $a$ such that $|u|_{a} \neq 0$. The path $u$ is closed (or a circuit) if $\alpha(u)=\omega(u)$. A circuit is simple if no proper subpath is a circuit. The graph $\Gamma$ is said to be strongly connected if, for any two vertices $v_{1}$ and $v_{2}$, there is a path from $v_{1}$ to $v_{2}$.

If $\Gamma=\langle V, E, \alpha, \omega\rangle$ is a graph, we denote by $\Gamma^{*}$ the free category generated by $\Gamma$, that is, the category whose set of vertices (or objects) is $V$ and whose set of edges (or morphisms) from a vertex $v_{1}$ to a vertex $v_{2}$ is the set of paths of $\Gamma$ whose initial and final vertices are $v_{1}$ and $v_{2}$, respectively.

For a finite graph $\Gamma$ and positive integers $m$ and $k$, let $\equiv_{m, k}$ be the congruence on $\Gamma^{*}$ such that, for coterminal edges $u, v$ of $\Gamma^{*}$,

$$
u \equiv_{m, k} v \quad \text { if }(\forall a \in E(\Gamma)),|u|_{a}=|v|_{a} \text { or }\left(|u|_{a},|v|_{a} \geqslant m \text { and }|u|_{a} \equiv|v|_{a}(\bmod k)\right) .
$$

Proposition 2.1. Let $\Gamma$ be a finite graph and $\sim$ a congruence of finite index over the free category $\Gamma^{*}$. Then $\Gamma^{*} / \sim$ belongs to $g \mathbf{C o m}_{m, k}$ if and only if $\equiv_{m, k} \subseteq \sim$.

Proof. Suppose $\equiv_{m, k} \subseteq \sim$ and let $F$ be the free monoid over $\mathbf{C o m}_{m, k}$ on $E(\Gamma)$. Then $F$ is a monoid of $\mathbf{C o m}_{m, k}$ and the projection from $\Gamma^{*} / \equiv_{m, k}$ onto $S$, which is the identity on edges and identifies all vertices, is faithful. Then $\Gamma^{*} / \equiv_{m, k}$ and, therefore, $\Gamma^{*} / \sim$ belong to $g \mathbf{C o m}_{m, k}$.

For the converse, suppose that $\Gamma^{*} / \sim$ belongs to $g \mathbf{C o m}_{m, k}$. Then there exists a monoid $S \in \mathbf{C o m}_{m, k}$ a category $T$, a quotient morphism $\delta: T \rightarrow \Gamma^{*} / \sim$, and a faithful morphism $\tau: E \rightarrow S$. Let $u=a_{1} \cdots a_{s}$ and $v=b_{1} \cdots b_{l}$, with $a_{1}, \ldots, a_{s}, b_{1}, \ldots, b_{l} \in E(\Gamma)$ and $u \equiv_{m, k} v$. For each $a \in\left\{a_{1}, \ldots, a_{s}, b_{1}, \ldots, b_{l}\right\}$ let $t_{a} \in T$ be such that $\delta\left(t_{a}\right)=a / \sim($ such a $t_{a}$ exists because $\delta$ is surjective on edges). Then $t_{a_{1}} \cdots t_{a_{s}}$ and $t_{b_{1}} \cdots t_{b_{l}}$ are paths in $T$, as $\delta$ is injective on vertices, and, as $\tau$ is faithful and $S \in \mathbf{C o m}_{m, k}, t_{a_{1}} \cdots t_{a_{s}}=t_{b_{1}} \cdots t_{b_{s}}$, and, consequently, $u \sim v$.

Proposition 2.1 gives an algorithm to decide whether a category belongs to $g \mathbf{C o m}_{m, k}$. Note that, by [15, Theorem 6.3] we already knew that $g \mathbf{C o m}_{m, k}$ and, by $[\mathbf{3}, \S 10.8]$ or $[\mathbf{1 0}], \mathbf{C o m}_{m, k} * \boldsymbol{D}$ and $\mathbf{C o m}_{m, k} * \boldsymbol{D}_{\ell}$ (with $\ell \geqslant 1$ ) are decidable. A more precise result is given below as Theorem 4.3.

The following result is fundamental in studying globals of pseudovarieties of commutative semigroups. A proof through the calculation of the semidirect product $\operatorname{Com} * \boldsymbol{D}$ is given in $[\mathbf{3}, \S 10.7]$.

Theorem 2.2 (Thérien and Weiss [16]). The pseudovariety $g$ Com is defined by the pseudoidentity

$$
\left(x y z=z y x ; \cdot \frac{x}{y} \cdot y^{y}\right) .
$$


For integers $m \geqslant 0$ and $k \geqslant 1$, denote by $M_{m, k}$ the monogenic monoid

$$
\left\langle a ; a^{m+k}=a^{m}\right\rangle
$$

(where $a^{0}$ is interpreted as being 1). Let $\boldsymbol{V}$ be a pseudovariety of semigroups. The ( Nelson) index of $\boldsymbol{V}$ is the largest non-negative integer $m$ such that $M_{m, 1} \in \boldsymbol{V}$ if the set of all such integers is bounded and is $\omega$ otherwise.

Recall that we denote by $\mathbb{N}$ the set of all non-negative integers. We define a realvalued function on $\mathbb{N} \times \mathbb{N}$ by letting $d(p, q)=2^{-r}$, where $r$ is the cardinality of the smallest monoid $M_{m, k}$ such that $a^{p} \neq a^{q}$ if there is such a monoid and taking $d(p, q)=0$ otherwise. Then it is well known and easy to see that $d$ is a metric on $\mathbb{N}$. We denote by $\hat{\mathbb{N}}$ the completion of this metric space, which, being in fact a projective limit of finite discrete sets, is compact. Note that the monoid $M_{m, k}$ is isomorphic to the additive semigroup of the semiring $\mathbb{N}_{m, k}$ of non-negative integers with threshold $m$ and period $k$. The composite of the mapping $p \mapsto a^{p}$ with this isomorphism is just the canonical projection $\mathbb{N} \rightarrow \mathbb{N}_{m, k}$, which is a semiring homomorphism for the usual addition and multiplication on $\mathbb{N}$. Hence $\mathbb{N}$ is in fact a metric semiring in the sense that its operations of addition and multiplication are uniformly continuous. Therefore, the completion $\hat{\mathbb{N}}$ inherits the structure of a semiring. By removing the additive neutral element 0 , we obtain the subsets $\mathbb{P}$ and $\hat{\mathbb{P}}$, respectively, from $\mathbb{N}$ and $\hat{\mathbb{N}}$.

For two elements $\pi$ and $\rho$ of $\hat{\mathbb{P}}$, we say that $\pi$ divides $\rho$ and we write $\pi \mid \rho$ if there is $\sigma \in \hat{\mathbb{P}}$ such that $\rho=\pi \sigma$. Moreover, since $\mathbb{P}$ is a lattice under division and the lattice operations gcd (greatest common divisor) and lcm (least common multiple) are uniformly continuous, $\hat{\mathbb{P}}$ is also a lattice under division whose gcd and lcm are continuous. Since $\hat{\mathbb{P}}$ is compact, any subset has a least upper bound (with respect to the division ordering). Hence, any subset of $\hat{\mathbb{P}}$ has a gcd and an $1 \mathrm{~cm}$. In particular, $\hat{\mathbb{P}}$ has an element $\omega$ which is a multiple of all other elements.

The elements of $\hat{\mathbb{P}} \backslash \mathbb{P}$ constitute an additive subgroup, namely the minimal ideal of the additive semigroup $\hat{\mathbb{P}}$. The neutral element of this group is precisely $\omega$ because, clearly, any $\pi \in \hat{\mathbb{P}}$ divides $\omega+\omega$ and so $\omega+\omega=\omega$. The additive inverse of $\omega+1$ in this group is then naturally denoted in the semigroup literature by $\omega-1$.

For $p \in \mathbb{P}$, denote by $p^{\omega}$ the $1 \mathrm{~cm}$ of all powers $p^{k}$ with $k \in \mathbb{P}$. From the uniqueness of factorization of integers in primes, we conclude that any $\pi \in \hat{\mathbb{P}}$ is the lcm of all $p^{k}$ dividing $\pi$, where $p$ runs over all primes and $k \in \mathbb{P} \cup\{\omega\}$.

To each $\pi \in \hat{\mathbb{P}}$, we associate a unary implicit operation on finite semigroups $x \mapsto x^{\pi}$ as follows. For a finite semigroup $S$ and an element $s \in S$, define $s^{\pi}$ to be $\hat{\varphi}(\pi)$, where $\hat{\varphi}$ is the unique continuous extension to $\hat{\mathbb{P}}$ of the homomorphism from the additive semigroup of $\mathbb{P}$ to $S$ which sends each $p$ to $s^{p}$. The correspondence between $\hat{\mathbb{P}}$ and the semigroup $\bar{\Omega}_{1} S$ of unary implicit operations is in fact a bijection and, moreover, addition in $\hat{\mathbb{P}}$ corresponds to pointwise multiplication of implicit operations while multiplication in $\hat{\mathbb{P}}$ corresponds to composition of implicit operations.

We define the period of a pseudovariety $\boldsymbol{V}$ of semigroups to be the lcm in $\hat{\mathbb{P}}$ of all positive integers $k$ such that $M_{0, k} \in \boldsymbol{V}$. 
From results of Nelson [14] and Almeida $[\mathbf{3}]$ it follows that the correspondence

$$
\begin{aligned}
(\mathbb{N} \cup\{\omega\}) \times \hat{\mathbb{P}} & \rightarrow \mathcal{P} s(\text { Com }), \\
(m, \pi) & \mapsto \mathbf{C o m}_{m, \pi}
\end{aligned}
$$

is a lattice embedding, where $\mathbb{N} \cup\{\omega\}$ is obtained from the chain $\mathbb{N}$ (under the usual order) by adding a maximum, $\hat{\mathbb{P}}$ is ordered by division, and $\mathcal{P} s(\mathbf{C o m})$ stands for the lattice of subpseudovarieties of Com.

We denote by $\bar{\Omega}_{\Gamma}$ Cat the free profinite category on a graph $\Gamma$ (cf. [9]), which may be viewed as the completion of the free category $\Gamma^{*}$ with respect to a natural metric associated with category homomorphisms (also known as functors) into finite categories. Recall that we view monoids as one-vertex categories by adding a virtual vertex. For a fixed edge $a \in E(\Gamma)$, since the composite of the mapping $\varphi: u \in E\left(\Gamma^{*}\right) \mapsto|u|_{a}$ with any projection $\mathbb{N} \rightarrow M_{m, k}$ defines a category homomorphism, $\varphi: E\left(\Gamma^{*}\right) \rightarrow \mathbb{N}$ is uniformly continuous and, therefore, extends uniquely to a continuous category homomorphism $\bar{\Omega}_{\Gamma}$ Cat $\rightarrow \hat{\mathbb{N}}$. The image $\hat{\varphi}(u)$ of an edge $u \in E\left(\bar{\Omega}_{\Gamma}\right.$ Cat $)$ is also denoted $|u|_{a}$.

Recall that a semigroupoid is an algebraic object like a category but without the requirement of local identities. The set of all loops at a vertex, if non-empty, forms a semigroup which is called a local semigroup of the semigroupoid. We say that a strongly connected semigroupoid $S$ has zeros if, for each pair of vertices $v_{1}, v_{2} \in V(S)$, there is an element $0_{v_{1}, v_{2}}$ such that, for every edge $s \in E(S)$, and every vertex $v \in V(S)$, the equalities $0_{v, \alpha(s)} s=0_{v, \omega(s)}$ and $s 0_{\omega(s), v}=0_{\alpha(s), v}$ hold. Note that, wherever they exist, zeros are unique.

A pseudovariety of semigroupoids is a class of finite semigroupoids containing the onevertex one-edge semigroupoid which is closed under divisors (in Tilson's sense [17]), and finite products and coproducts. A pseudovariety of semigroupoids is said to be categorical if it is generated by its categories. For a pseudovariety $\boldsymbol{V}$ of semigroups, $\ell \boldsymbol{V}$ denotes the pseudovariety of semigroupoids consisting of all finite semigroupoids whose local semigroups lie in $\boldsymbol{V}$.

\section{The vertex rank}

For integers $r$ and $m$ greater than 1, let $L_{r, m}$ be the locally commutative category with zeros generated by the graph $G_{r}$ described by the diagram

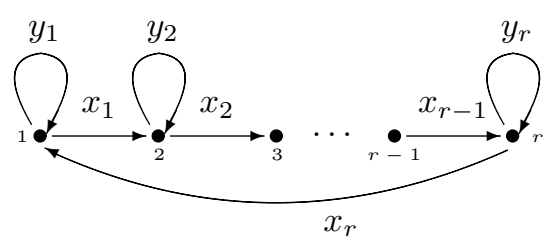

subject to the relations

$$
\begin{aligned}
y_{i}^{2} & =0_{i, i}, \\
x_{i} \cdots x_{r}\left(x_{1} \cdots x_{r}\right)^{m-2} x_{1} \cdots x_{i+1} & =0_{i, i+2},
\end{aligned}
$$




$$
\begin{aligned}
& z 0_{\omega(z), j}=0_{\alpha(z), j}, \\
& 0_{i, \alpha(z)} z=0_{i, \omega(z)},
\end{aligned}
$$

where $z$ denotes an arbitrary edge of the graph $G_{r}$.

Lemma 3.1. The category $L_{r, m}$ has the following properties:

(i) $L_{r, m} \in g$ Com;

(ii) if $w$ is a path in $G_{r}$ representing an edge of $L_{r, m}$ such that $|w|_{a}>m$ for some edge $a$ of the graph $G_{r}$, then $w=0_{\alpha(w), \omega(w)}$ in $L_{r, m}$.

Proof. (i) By Theorem 2.2, it suffices to show that $L_{r, m}$ satisfies the pseudoidentity (2.1). So, suppose $x, y$ and $z$ are three paths in the graph $G_{r}$ with common ends as in (2.1). By local commutativity, if an edge $y_{i}$ is at all used in the path $x y z$ (or, equivalently, in the path $z y x$ ), then we may pull, in both paths $x y z$ and $z y x$, the edge $y_{i}$ to the first (perhaps only) time the path goes through the vertex $i$, without thus changing the value of the two paths in $L_{r, m}$. Then what remains in the two paths are the edges $x_{i}$, which constitute a cycle. Therefore, the value of the path depends only on where it starts, where it ends, and how many times it goes through each edge $x_{i}$. Since these parameters are the same for the paths $x y z$ and $z y x$, it follows that $x y z=z y x$. Hence $L_{r, m} \in g$ Com.

(ii) If some edge $y_{i}$ is used more than once in $w$, then local commutativity and the relations defining $L_{r, m}$ imply that $w$ is a zero in $L_{r, m}$. So, suppose $w$ goes through an edge $x_{i}$ at least $m+1$ times. Then $w$ must contain $m$ subpaths from the vertex $i+1$ to the vertex $i$. Using local commutativity to pull all occurrences of edges $y_{j}$ to the first such subpath, we conclude that $w$ may be factorized in $L_{r, m}$ so as to contain a factor of the form

$$
\begin{aligned}
\left(x_{i} x_{i+1} \cdots x_{r} x_{1} \cdots x_{i-1}\right)^{m} x_{i} & =x_{i} \cdots x_{r}\left(x_{1} \cdots x_{r}\right)^{m-2} x_{1} \cdots x_{i+1} x_{i+2} \cdots x_{r} x_{1} \cdots x_{i} \\
& =0_{i, i+2} x_{i+2} \cdots x_{r} x_{1} \ldots x_{i}=0_{i, i}
\end{aligned}
$$

which establishes the claim that $w$ is a zero in $L_{r, m}$.

Consider the following pseudoidentity $\varepsilon_{r, m, \pi}$ over the graph $G_{r}$ :

$$
y_{1} x_{1} y_{2} \cdots x_{r-1} y_{r} x_{r}\left(x_{1} \cdots x_{r}\right)^{m-1+\pi}=y_{1} x_{1} y_{2} \cdots x_{r-1} y_{r} x_{r}\left(x_{1} \cdots x_{r}\right)^{m-1} .
$$

It plays an important role in the sequel. Note that it holds in $\mathbf{C o m}_{m, \pi}$ and, therefore, also in $g \mathbf{C o m}_{m, \pi}$.

Proposition 3.2. The category $L_{r, m}$ belongs to $g \mathbf{C o m}_{m+1,1}$ but not to $g \mathbf{C o m}_{m, \omega}$.

Proof. As observed above, the pseudoidentity $\varepsilon_{r, m, \omega}$ holds in $g \mathbf{C o m}_{m, \omega}$. The lefthand side of $\varepsilon_{r, m, \omega}$ represents the element $0_{1,1}$ of $L_{r, m}$, whereas the right-hand side does not. Hence $L_{r, m} \notin g \mathbf{C o m}_{m, \omega}$.

It remains to show that $L_{r, m} \in g \mathbf{C o m}_{m+1,1}$. Suppose that $(u=v ; \Gamma)$ is a pseudoidentity over a finite graph $\Gamma$ which holds in $g \mathbf{C o m}_{m+1,1}$. If, for every $a \in E(\Gamma),|u|_{a}=$ 
$|v|_{a}$, then $(u=v ; \Gamma)$ holds in Com and, therefore, in all of $g$ Com. By Lemma 3.1 (i), it then follows that $L_{r, m}$ satisfies $(u=v ; \Gamma)$. On the other hand, if $|u|_{a} \neq|v|_{a}$ for some $a \in E(\Gamma)$, then $|u|_{a}$ and $|v|_{a}$ must both be at least $m+1$. Then by Lemma 3.1 (ii), both sides of the pseudoidentity $(u=v ; \Gamma)$ evaluate to the same $0_{i, j}$. This establishes the claim that $L_{r, m} \in g \mathbf{C o m}_{m+1,1}$.

We next prove the following critical property of $L_{r, m}$ with respect to the pseudovariety $g \mathbf{C o m}_{m, 1}$.

Proposition 3.3. For each integer $m \geqslant 2$, every subcategory of $L_{r, m}$ on at most $r-1$ vertices lies in the global $g \mathbf{C o m}_{m, 1}$.

Proof. Let $(u=v ; \Gamma)$ be a pseudoidentity over a finite graph $\Gamma$ which holds in the global $g \mathbf{C o m}_{m, 1}$ and let $C$ be a subcategory of $L_{r, m}$ with at most $r-1$ vertices. We want to show that $C$ satisfies $(u=v ; \Gamma)$. In view of the symmetry of the graph $G_{r}$, it suffices to consider the case of the subcategory $C$ generated by the following graph:

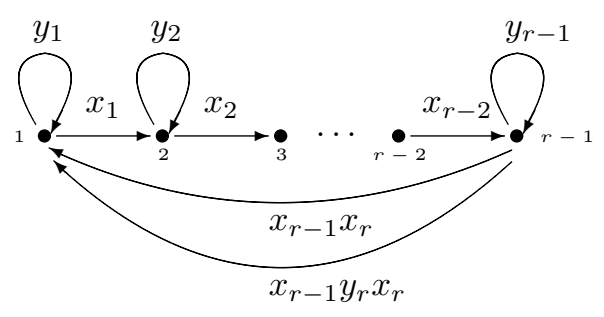

The verification of $(u=v ; \Gamma)$ in $C$ is similar to the second part of the proof of Proposition 3.2. Indeed, if $|u|_{a} \neq|v|_{a}$ for some $a \in E(\Gamma)$, then $|u|_{a}$ and $|v|_{a}$ are both at least $m$. If, after evaluation in $C, u$ and $v$ are not both zeros, $a$ must then be evaluated to a path in the $x_{i}(i=1, \ldots, r-2)$ and $x_{r-1} x_{r}$. Since $|u|_{a},|v|_{a} \geqslant m$, both $u$ and $v$ must contain at least $m-1$ factors $w$ such that $\alpha(w)=\omega(a)$ and $\omega(w)=\alpha(a)$. By the assumption that $|u|_{a}$ and $|v|_{a}$ are distinct and both greater than or equal to $m$, it follows from Lemma 3.1 (ii) that we may assume that, under evaluation of $\Gamma$ in $C, v$ evaluates to a zero. Assuming $u$ does not also evaluate to a zero, then $|u|_{b} \leqslant m$ for every $b \in E(\Gamma)$. Moreover, as above, $v$ must contain at least $m$ factors $w$ such that $\alpha(w)=\omega(a)$ and $\omega(w)=\alpha(a)$. In view of the structure of the graph (3.1), this implies that, after evaluation of $\Gamma$ in $C,|v|_{x_{i}} \geqslant m$ for all $i$ and so also $|u|_{x_{i}} \geqslant m$ for all $i$. Since, by Lemma 3.1 (ii), no $x_{i}$ can occur more than $m$ times in a path which is non-zero in $L_{r, m}$, we conclude that $u$ must evaluate in $C$ to a circuit of the form

$$
y_{i} x_{i} \cdots x_{r-1} y_{r} x_{r} y_{1} x_{1} \cdots y_{i-1} x_{i-1}\left(x_{i} \cdots x_{r} x_{1} \cdots x_{i-1}\right)^{m-1}
$$

with $i \neq r$, while $v$ evaluates to $0_{i, i}$. Since $(u=v ; \Gamma)$ is assumed to be valid in $g \mathbf{C o m}_{m, 1}$, the value $0_{i, i}$ for $v$ must be obtained as the value in $L_{r, m}$ of a circuit of the form

$$
y_{i} x_{i} \cdots x_{r-1} y_{r} x_{r} y_{1} x_{1} \cdots y_{i-1} x_{i-1}\left(x_{i} \cdots x_{r} x_{1} \cdots x_{i-1}\right)^{m} .
$$


But in $C$, the $L_{r, m}$ product $x_{r-1} x_{r}$ cannot be split into factors, which implies that the edge $b$ whose evaluation in $C$ produces $x_{r-1} x_{r}$ as a factor is such that $|u|_{b}<m$ while $|v|_{b} \geqslant m$ : a contradiction since the value of $u$ in $C$ is then also a zero. This shows that $C$ satisfies the pseudoidentity $(u=v ; \Gamma)$ and completes the proof that $C \in g \mathbf{C o m}_{m, 1}$.

We may now prove the main result of this section, which, in particular, in view of the results of [10], provides a negative solution to problems (40) and (41) from [3].

Theorem 3.4. For any integer $m \geqslant 2$, no pseudovariety $\boldsymbol{V}$ between $g \mathbf{C o m}_{m, 1}$ and $g \mathbf{C o m}_{m, \omega}$ has finite vertex rank.

Proof. Arguing by contradiction, suppose such a pseudovariety $\boldsymbol{V}$ admits a basis $\Sigma$ over graphs with at most $r$ vertices. We claim that $L_{r+1, m}$ must then lie in $\boldsymbol{V}$. Indeed, in verifying the pseudoidentities of $\Sigma$, we only consider subcategories of $L_{r+1, m}$ in at most $r$ vertices and, by Proposition 3.3, such subcategories lie in $g \mathbf{C o m}_{m, 1}$ and, therefore, also in $\boldsymbol{V}$. Since $\boldsymbol{V} \subseteq g \mathbf{C o m}_{m, \omega}$, it follows that $L_{r+1, m} \in g \mathbf{C o m}_{m, \omega}$, in contradiction with Proposition 3.2.

Further properties of the categories $L_{r, m}$ are given in $\S 5.4$.

Note that the interval of category pseudovarieties $\left[g \mathbf{C o m}_{m, 1}, g \mathbf{C o m}_{m, \omega}\right]$ is uncountable as it contains each of the pseudovarieties $g \mathbf{C o m}_{m, \pi}$ with $\pi \in \hat{\mathbb{P}}$, different values of $\pi$ corresponding to different pseudovarieties.

Theorem 3.5. For $m \in\{0,1, \omega\}$ and $\pi \in \hat{\mathbb{P}}$, the pseudovariety $g \mathbf{C o m}_{m, \pi}$ is one of the following:

(a) $\llbracket(x y=y x ; \bigcirc x, y),\left(x^{\pi} y=y ; \stackrel{x}{C} \cdot \stackrel{y}{\rightarrow} \cdot\right),\left(y x^{\pi}=y ; \stackrel{y}{\rightarrow} \bigcirc^{x}\right) \rrbracket$ in the case $m=0$

(b) $\llbracket(x y=y x ; \bullet x, y),\left(x^{1+\pi}=x ; \bullet x\right) \rrbracket$ in the case $m=1$;

(c) $g \operatorname{Com} \cap \llbracket\left(x^{\omega+\pi}=x^{\omega} ; \bullet x\right) \rrbracket$ in the case $m=\omega$.

In particular, in all cases $g \mathbf{C o m}_{m, \pi}$ is finitely based with vertex rank at most 2 .

Proof. In case $m=0$, the result follows from the locality of the group pseudovariety $\mathbf{C o m}_{0, \pi}$ as a pseudovariety of categories $[\mathbf{1 5}, \mathbf{1 7}]$ together with the remarks at the end of $\S 2$ in $[\mathbf{9}]$.

In the case $m=1$, the pseudovariety $\mathbf{C o m}_{1, k}$ is local for $k \in \mathbb{P}$, i.e. $\mathbf{C o m}_{1, k} * \boldsymbol{D}=$ $\mathcal{L C o m}_{1, k}[\mathbf{3}$, Corollary 10.8.4] (cf. the Straubing-Tilson Delay Theorem [17], where, for a pseudovariety $\boldsymbol{V}$ of semigroups, $\mathcal{L} \boldsymbol{V}$ denotes the pseudovariety consisting of all finite semigroups $S$ whose subsemigroups of the form $e S e$, with $e^{2}=e \in S$, all lie in $\boldsymbol{V}$ ). Since

$$
\mathbf{C o m}_{m, \pi}=\bigcup_{k \in \mathbb{P}, k \mid \pi} \mathbf{C o m}_{m, k}
$$

it follows that $\mathbf{C o m}_{1, \pi} * \boldsymbol{D}=\mathcal{L} \mathbf{C o m}_{1, \pi}$ for every $\pi \in \hat{\mathbb{P}}$. Hence $\mathbf{C o m}_{1, \pi}$ is local. 
In the case $m=\omega,[\mathbf{3}$, Corollary 10.7.8] shows that

$$
\mathbf{C o m}_{\omega, k} * \boldsymbol{D}=(\mathbf{C o m} * \boldsymbol{D}) \cap \llbracket x^{\omega+k}=x^{\omega} \rrbracket
$$

for $k \in \mathbb{P}$. As in the previous case, this implies that the same equality holds for all $k \in \hat{\mathbb{P}}$. By, for example, the results of [10] and Theorem 2.2, it follows that the global $g \mathbf{C o m} \mathbf{o m}_{m, \pi}$ is given by (c).

\section{Decidability}

To investigate the decidability of pseudovarieties of the form $g \mathbf{C o m}_{m, \pi}$, we first exhibit an infinite basis of pseudoidentities for each of these pseudovarieties in the case where $m$ is an integer greater than 1 .

Theorem 4.1. For every integer $m \geqslant 2$ and every $\pi \in \hat{\mathbb{P}} \backslash \mathbb{P}$, the pseudovariety $g \mathbf{C o m}_{m, \pi}$ is the intersection of $g \mathbf{C o m}$ with the pseudovariety defined by the sequence of pseudoidentities $\left(\varepsilon_{r, m, \pi}\right)_{r \geqslant 1}$ taken as pseudoidentities of categories, i.e. any of the $y_{i}$ may be erased.

Proof. That $g \mathbf{C o m}_{m, \pi}$ is contained in the intersection is immediate since the operator $g$ respects the inclusion ordering. For the reverse inclusion, since $g \mathbf{C o m}_{m, \pi}$ is a nontrivial categorical pseudovariety of semigroupoids, it is defined by pseudoidentities over strongly connected graphs [9]. Consider a strongly connected pseudoidentity of categories $(u=v ; \Gamma)$ which holds in $g \mathbf{C o m}_{m, \pi}$. It suffices to show that, for every positive integer $k$ dividing $\pi$,

$$
g \operatorname{Com} \cap \llbracket \varepsilon_{r, m, k}: r \geqslant 2 \rrbracket \models(u=v ; \Gamma) .
$$

The condition $g \mathbf{C o m}_{m, \pi} \models(u=v ; \Gamma)$ means that, for every $a \in E(\Gamma),|u|_{a}=|v|_{a}$ in $\left(\bar{\Omega}_{a} \mathbf{C o m}_{m, \pi}\right)^{1}$. If actually $|u|_{a}=|v|_{a}$ in $\hat{\mathbb{N}}$ for every $a \in E(\Gamma)$, then $g$ Com satisfies $(u=v ; \Gamma)$, which implies (4.1). Otherwise, there is at least one edge $a \in E(\Gamma)$ such that $|u|_{a} \neq|v|_{a}$ in $\hat{\mathbb{N}}$ and so $|u|_{a}$ and $|v|_{a}$ do not both belong to $\mathbb{N}$.

To proceed we need the following combinatorial lemma.

Lemma 4.2. Let $\Gamma$ be a finite graph and let $a \in E(\Gamma)$ and $w \in E\left(\bar{\Omega}_{\Gamma} \mathbf{C a t}\right)$ be such that $|w|_{a} \notin \mathbb{N}$. Then there is a circuit $\gamma$ in $\Gamma$ containing the edge a such that, for every $b \in E(\gamma),|w|_{b} \notin \mathbb{N}$.

Proof. Since the result is obvious if $a$ is a loop in $\Gamma$, we assume that $a$ is not a loop.

Let $\left(w_{n}\right)_{n}$ be a sequence of paths of $\Gamma$ converging to $w$ in the profinite topology. Arguing by contradiction, suppose that every circuit $\gamma$ containing the edge $a$ has some edge $b$ such that $|w|_{b} \in \mathbb{N}$. Since the graph $\Gamma$ is finite, there are only finitely many simple circuits $\gamma_{1}, \ldots, \gamma_{r}$ containing $a$. For each of these circuits $\gamma_{i}$, let $b_{i} \in E\left(\gamma_{i}\right)$ be such that $|w|_{b_{i}} \in \mathbb{N}$. By taking a subsequence of $\left(w_{n}\right)_{n}$, we may further assume that $\left|w_{n}\right|_{b_{i}}=|w|_{b_{i}}$ for $i=1, \ldots, r$ and all $n$. Since $|w|_{a} \notin \mathbb{N}$, there is some $n$ such that $\left|w_{n}\right|_{a}=m$ with $m>1+|w|_{b_{1}}+\cdots+|w|_{b_{r}}$. Then $w_{n}$ is a path in the graph $\Gamma$ which goes through the edge $a$ precisely $m$ times and, therefore, includes $m-1$ subpaths from $\omega(a)$ to $\alpha(a)$. From 
each such subpath, we may extract a path which, together with the edge $a$, completes one of the circuits $\gamma_{1}, \ldots, \gamma_{r}$. Hence $w_{n}$ should go through the edges $b_{1}, \ldots, b_{r}$ a total of at least $m-1$ times, in contradiction with the above choices. This shows that there must exist a circuit $\gamma$ as claimed.

Returning to the proof of Theorem 4.1, next we consider sequences of paths $\left(u_{n}\right)_{n}$ and $\left(v_{n}\right)_{n}$ which are coterminal with $u$ and $v$ and converge, respectively, to $u$ and $v$ in the profinite topology. Since $\bar{\Omega}_{a} \mathbf{C o m}_{m, k}$ is finite, by taking subsequences we may assume that $\left|u_{n}\right|_{a}=|u|_{a}$ and $\left|v_{n}\right|_{a}=|v|_{a}$ in $\bar{\Omega}_{a} \mathbf{C o m}_{m, k}$ for all $n$ and all $a \in E(\Gamma)$. We may further assume that $\left|u_{n}\right|_{a}=|u|_{a}$ for all $n$ whenever $|u|_{a} \in \mathbb{N}$, and that $\left|u_{n}\right|_{a} \geqslant m+1$ for all $n$ whenever $|u|_{a} \notin \mathbb{N}$. Similar assumptions may be forced to hold for the pair $\left(\left(v_{n}\right)_{n}, v\right)$. If $\left|u_{n}\right|_{a} \neq\left|v_{n}\right|_{a}$ for a certain $n$, it then follows that at least one of $|u|_{a}$ and $|v|_{a}$ does not belong to $\mathbb{N}$. By Lemma 4.2, we deduce that every edge $a \in E(\Gamma)$ such that $\left|u_{n}\right|_{a} \neq\left|v_{n}\right|_{a}$ is part of a circuit $\gamma$ such that either for every $b \in E(\gamma),|u|_{b} \notin \mathbb{N}$ or for every $b \in E(\gamma),|v|_{b} \notin \mathbb{N}$. Since $k$ divides $\pi$, in this way we guarantee that, for every $n$,

(i) for every $a \in E(\Gamma),\left|u_{n}\right|_{a} \equiv_{m, k}\left|v_{n}\right|_{a}$;

(ii) if $\left|u_{n}\right|_{a} \neq\left|v_{n}\right|_{a}$, then there is a cycle $\gamma$ in $\Gamma$ containing the edge $a$ such that, for every $b \in E(\gamma),\left|u_{n}\right|_{b},\left|v_{n}\right|_{b} \geqslant m$.

By [3, Corollary 5.7.4], it is possible to transform to the same array of non-negative integers each of the arrays $\left(\left|u_{n}\right|_{a}\right)_{a \in E(\Gamma)}$ and $\left(\left|v_{n}\right|_{a}\right)_{a \in E(\Gamma)}$ by finite sequences of operations which, for cycles for which all of the corresponding components are at least $m$, add $k$ to all such components. Now, in $g \mathbf{C o m}$, the value of a path in the graph $\Gamma$ depends only on where it starts, where it ends, and how many times it goes through each edge. So, if a path $w$ goes through all edges of a cycle $\gamma$ at least $m$ times, and the cyclic order of the edges of the path is $x_{1}, \ldots, x_{r}$, then, up to equality in $g \mathbf{C o m}, w$ has a factor of the form

$$
y_{1} x_{1} y_{2} \cdots x_{r-1} y_{r} x_{r}\left(x_{1} \cdots x_{r}\right)^{m-1},
$$

where, without loss of generality, $i=1$ is assumed to be such that $\alpha\left(x_{i}\right)$ is at minimum distance from $\alpha(w)$ in $\Gamma$. Using the pseudoidentity $\varepsilon_{r, m, k}$, it follows that the operation of adding $k$ to each $|w|_{x_{i}}$, leaving unchanged the values of all other $|w|_{a}(a \in E(\Gamma) \backslash$ $\left.\left\{x_{1}, \ldots, x_{r}\right\}\right)$, is such that any path $w^{\prime}$ which has the same $\left|w^{\prime}\right|_{a}$ values is such that

$$
g \operatorname{Com} \cap \llbracket \varepsilon_{r, m, k}: r \geqslant 2 \rrbracket \models\left(w=w^{\prime} ; \Gamma\right) .
$$

This proves (4.1) and completes the proof of Theorem 4.1.

We may now establish the following decidability criterion for globals of pseudovarieties of the form $g \mathbf{C o m}_{m, \pi}$.

Theorem 4.3. For $m \in \mathbb{N} \cup\{\omega\}, \pi \in \hat{\mathbb{P}}$, the following conditions are equivalent:

(i) the pseudovariety of semigroupoids $g \mathbf{C o m}{ }_{m, \pi}$ is decidable;

(ii) the pseudovariety of semigroups $\mathbf{C o m} m, \pi$ is decidable; 
(iii) it is decidable when a positive integer $k$ divides $\pi$.

Proof. (i) $\Rightarrow$ (ii) In general, since a pseudovariety $\boldsymbol{V}$ of semigroups consists precisely of those semigroups which lie in $g \boldsymbol{V}$, if $g \boldsymbol{V}$ is decidable then so is $\boldsymbol{V}$.

(ii) $\Rightarrow$ (iii) Let $k$ be a positive integer. Then the cyclic group $M_{0, k}$ of order $k$ belongs to $\mathbf{C o m}_{m, \pi}$ if and only if $k$ divides $\pi$. Hence, assuming (ii) we deduce (iii).

(iii) $\Rightarrow$ (i) Let $S$ be a finite semigroupoid. Assuming (iii), we show that there is an algorithm to test whether $S \in g \mathbf{C o m}_{m, \pi}$.

Suppose first that $m$ is an integer greater than 1 . If $\pi \in \mathbb{P}$, we have already observed that $g \mathbf{C o m}_{m, \pi}$ is decidable. So, assume $\pi \notin \mathbb{P}$. In view of Theorems 4.1 and 2.2 , it suffices to show that it is decidable whether a semigroupoid $S \in g$ Com satisfies all pseudoidentities $\varepsilon_{r, m, \pi}$ with $r \geqslant 1$. Let $r>|E(S)|$. By the pigeon-hole principle, in any evaluation of the graph $G_{r}$ in $S$, two of the edges $x_{i}$ and $x_{j}$ with $i<j$ must be mapped to the same edge. This means that the cycle $x_{1} \ldots x_{r}$ maps to the union of two circuits with at least one common edge. Since $S \in g \mathbf{C o m}$, the change in the values $|\cdot|_{x_{i}}$ (adding $\pi$ ) may then be performed separately in each of these circuits provided $S$ satisfies all pseudoidentities $\varepsilon_{s, m, \pi}$ with $s<r$. Hence $S$ satisfies all pseudoidentities $\varepsilon_{r, m, \pi}(r \geqslant 1)$ if and only if it satisfies all $\varepsilon_{r, m, \pi}$ with $1 \leqslant r \leqslant|E(S)|$.

So, in the case where $m$ is an integer greater than 1 and $\pi \in \hat{\mathbb{P}} \backslash \mathbb{P}$, it remains to show that it is decidable whether a finite semigroupoid $S$ satisfies the pseudoidentity $\varepsilon_{r, m, \pi}$ for a given $r \geqslant 1$. This amounts to showing that there is an algorithm to compute, for a given finite semigroupoid $S$ and a given loop $w \in S$, the power $w^{\pi}$. In view of Theorem 3.5, this is also all that needs to be done in case $m \in\{0,1, \omega\}$. Since $w^{\pi}$ lies in the cyclic local subsemigroup generated by $w$, it suffices now to invoke well-known facts about unary implicit operations, as may be found, for example, in $[\mathbf{3}, \S 3.7]$.

\section{Gaps}

In this section, we study the gaps in the skeleton of subpseudovarieties of $g$ Com consisting of the pseudovarieties of the form $g \mathbf{C o m}_{m, k}$ with $m \in \mathbb{P} \cup\{0\}$ and $k \in \mathbb{P}$.

\subsection{The categories $C_{n, m, k}$ and $D_{n, m, k}$}

Fix integers $n, m, k \geqslant 1$ and denote by $A_{n}$ the graph
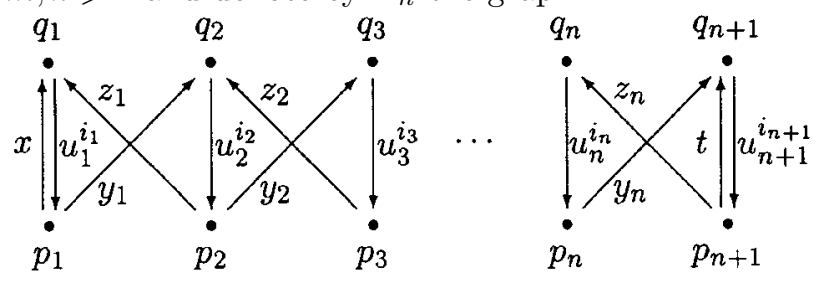

where the notation $u_{j}^{i_{j}}$ is meant to indicate, for each lower index $j$, one of several coterminal edges with the upper index running over the corresponding set as follows: $i_{1}, i_{n+1} \in\{1,2, \ldots, 2 m+k\}$ and, for $j \notin\{1, n+1\}, i_{j} \in\{1,2, \ldots, 2 m+k+1\}$. For simplicity we will sometimes write $z_{0}$ or $y_{0}$ for $x$, and $z_{n+1}$ or $y_{n+1}$ for $t$. 
Consider the free category $A_{n}^{*}$ and the following two relations, $\sim_{n, m, k}$ and $\approx_{n, m, k}$, on $A_{n}^{*}$. For coterminal edges $v, w$ of $A_{n}^{*}$, set

(i) $v \sim_{n, m, k} w$ if $\left\{\begin{array}{l}(\exists i, l)|v|_{u_{i}^{l}} \geqslant 2 \text { and }(\exists i, l)|w|_{u_{i}^{l}} \geqslant 2 \\ \text { or } \\ \left(\forall a \in E\left(A_{n}\right)\right)|v|_{a}=|w|_{a} ;\end{array}\right.$
(ii) $v \approx_{n, m, k} w$ if $\left\{\begin{array}{l}(\exists i, l)|v|_{u_{i}^{l}} \geqslant 2 \text { and }(\exists i, l)|w|_{u_{i}^{l}} \geqslant 2 \\ \text { or } \\ v \equiv_{m, k} w .\end{array}\right.$

Note that both $\sim_{n, m, k}$ and $\approx_{n, m, k}$ are congruences on $A_{n}^{*}$.

Let $C_{n, m, k}$ and $D_{n, m, k}$ denote, respectively, the quotient categories $A_{n}^{*} / \sim_{n, m, k}$ and $A_{n}^{*} / \approx_{n, m, k}$. By definition, $D_{n, m, k}$ is a quotient of $C_{n, m, k}$ and, as $\sim_{n, m, k}$ and $\approx_{n, m, k}$ are finite index congruences, $C_{n, m, k} \in g \mathbf{C o m}$ and, by Proposition 2.1, $D_{n, m, k} \in g \mathbf{C o m}_{m, k}$. Note that an element $v \in E\left(A_{n}^{*}\right)$ such that $|v|_{u_{i}^{l}} \geqslant 2$ for some $i, l$ is a zero element, the $\sim_{n, m, k^{-}}$and $\approx_{n, m, k^{-}}$classes of such elements being precisely the local zeros, respectively, of $A_{n}^{*} / \sim_{n, m, k}$ and $A_{n}^{*} / \approx_{n, m, k}$. We will prove that $C_{n, m, k} \notin g \mathbf{C o m}_{m, k}$, but, for $m>1$, every subcategory $B$ of $C_{n, m, k}$ such that $V(B) \neq V\left(C_{n, m, k}\right)$, belongs to $g \mathbf{C o m}_{m, k}$.

In this subsection we establish some results concerning the congruences $\sim_{n, m, k}$ and $\approx_{n, m, k}$. We adopt the following simplifying notational conventions:

(i) for a non-zero edge $v \in E\left(A_{n}^{*}\right)$ and $i \in\{1, \ldots, n+1\}$, let $|v|_{i}$ denote the number of upper indices $l$ such that $|v|_{u_{i}^{l}}=1$;

(ii) we will write, sometimes, $u_{i}$ for an edge of the form $u_{i}^{j}$; we write, for example, $\left(z_{1} u_{1} y_{1} u_{2}\right)^{r}$ to mean a product of $r$ edges of the form $z_{1} u_{1}^{i} y_{1} u_{2}^{j}$ with no edges in common other than $z_{1}$ and $y_{1}$.

We start with an easy observation, which separates the case $m=1$ from $m>1$.

Lemma 5.1. Let $B$ be a subcategory of $C_{n, m, k}$ and suppose that $w$ is a cycle in $B$ that is neither a zero nor an identity. Then, for every $l>1, w^{l} \neq w$. In particular $B \notin \ell \mathbf{C o m}_{1, k}$ and, consequently, $B \notin g \mathbf{C o m}_{1, k}$.

Proof. Just note that $w^{l}$ is a local zero and so $w^{l}$ is different from $w$. In particular $w^{1+k} \neq w$, which proves that $B \notin \ell \mathbf{C o m}_{1, k}$.

Next we give a nice necessary and sufficient condition for two elements of $A_{n}^{*}$ to be $\sim_{n, m, k}$-equivalent. For this we need some preliminary results.

Lemma 5.2. Let $w \in E\left(A_{n}^{*}\right)$. Then, for all $j \in\{1, \ldots, n+1\}$,

$$
|w|_{j}-\left(|w|_{z_{j-1}}+|w|_{y_{j}}\right)= \begin{cases}0 & \text { if } \alpha(w) \neq \omega\left(u_{j}\right) \neq \omega(w), \\ 1 & \text { if } \alpha(w) \neq \omega\left(u_{j}\right)=\omega(w) \\ -1 & \text { if } \alpha(w)=\omega\left(u_{j}\right) \neq \omega(w) \\ 0 & \text { if } \alpha(w)=\omega\left(u_{j}\right)=\omega(w)\end{cases}
$$


and

$$
|w|_{j}-\left(|w|_{z_{j}}+|w|_{y_{j-1}}\right)= \begin{cases}0 & \text { if } \alpha(w) \neq \alpha\left(u_{j}\right) \neq \omega(w) \\ -1 & \text { if } \alpha(w) \neq \alpha\left(u_{j}\right)=\omega(w) \\ 1 & \text { if } \alpha(w)=\omega\left(u_{j}\right) \neq \omega(w) \\ 0 & \text { if } \alpha(w)=\alpha\left(u_{j}\right)=\omega(w)\end{cases}
$$

In particular, $|w|_{j}-\left(|w|_{z_{j-1}}+|w|_{y_{j}}\right)$ and $|w|_{j}-\left(|w|_{z_{j}}+|w|_{y_{j-1}}\right)$ depend only on the initial and terminal vertices of $w$.

Corollary 5.3. Let $v$ and $w$ be non-zero coterminal edges of $A_{n}^{*}$ with the same content. If $1 \leqslant j \leqslant n+1$, then

$$
\begin{aligned}
|v|_{z_{j}} & =|w|_{z_{j}} \Leftrightarrow|v|_{y_{j-1}}=|w|_{y_{j-1}}, \\
|v|_{y_{j}} & =|w|_{y_{j}} \Leftrightarrow|v|_{z_{j-1}}=|w|_{z_{j-1}} .
\end{aligned}
$$

Proof. As $v$ and $w$ are coterminal we have, applying the previous lemma to $v$ and $w$,

$$
\begin{aligned}
|v|_{j}-\left(|v|_{z_{j-1}}+|v|_{y_{j}}\right) & =|w|_{j}-\left(|w|_{z_{j-1}}+|w|_{y_{j}}\right), \\
|v|_{j}-\left(|v|_{z_{j}}+|v|_{y_{j-1}}\right) & =|w|_{j}-\left(|w|_{z_{j}}+|w|_{y_{j-1}}\right) .
\end{aligned}
$$

As $v$ and $w$ have the same content and are non-zero, $|v|_{j}=|w|_{j}$. Hence, $|v|_{z_{j-1}}+|v|_{y_{j}}=$ $|w|_{z_{j-1}}+|w|_{y_{j}}$ and $|v|_{z_{j}}+|v|_{y_{j-1}}=|w|_{z_{j}}+|w|_{y_{j-1}}$, from which the result follows.

We are now ready to give a characterization of the congruence $\sim_{n, m, k}$.

Proposition 5.4. Let $v$ and $w$ be non-zero coterminal edges of $A_{n}^{*}$ with the same content. Then, the following conditions are equivalent:

(i) $v \sim_{n, m, k} w$;

(ii) there exists $s \in\{0,1, \ldots, n+1\}$ such that $|v|_{z_{s}}=|w|_{z_{s}}$;

(iii) there exists $s \in\{0,1, \ldots, n+1\}$ such that $|v|_{y_{s}}=|w|_{y_{s}}$.

Proof. We prove that (ii) implies (i).

By definition of $\sim_{n, m, k}$, as $v$ and $w$ are non-zero coterminal edges with the same content, we need to prove that, for every $a \in c(v),|v|_{a}=|w|_{a}$. If $a$ is of the form $u_{j}^{l}$, then $|v|_{u_{j}^{l}}=|w|_{u_{j}^{l}}=1$. It remains to prove that, for every $j,|v|_{z_{j}}=|w|_{z_{j}}$ and $|v|_{y_{j}}=|w|_{y_{j}}$.

Using Corollary 5.3 we see that, for every $j \in\{0,1, \ldots, n+1\},|v|_{z_{j}}=|w|_{z_{j}}$ or $|v|_{y_{j}}=$ $|w|_{y_{j}}$. Then, again using Corollary 5.3, as $x=y_{0}=z_{0},|v|_{x}=|w|_{x}$, it follows that, for every $j \in\{0,1, \ldots, n+1\},|v|_{z_{j}}=|w|_{z_{j}}$ and $|v|_{y_{j}}=|w|_{y_{j}}$, which proves that $v \sim_{n, m, k}$ $w$.

Corollary 5.5. Let $v$ and $w$ be non-zero coterminal edges of $A_{n}^{*}$. If $v \approx_{n, m, k} w$ and there exists $i$ such that $|v|_{z_{i}}<m$ or $|v|_{y_{i}}<m$, then $v \sim_{n, m, k} w$. 
Proof. Suppose $|v|_{z_{i}}<m$. As $v \approx_{n, m, k} w,|v|_{z_{i}} \equiv_{m, k}|w|_{z_{i}}$ and, as $|v|_{z_{i}}<m,|v|_{z_{i}}=$ $|w|_{z_{i}}$. By Proposition 5.4, we deduce that $v \sim_{n, m, k} w$.

We next note that $C_{n, m, k} \notin g \mathbf{C o m}_{m, k}$. This is a simple observation and could have been made immediately after the definition of $C_{n, m, k}$.

Proposition 5.6. The category $C_{n, m, k}$ does not belong to $g \mathbf{C o m}_{m, k}$.

Proof. Using Proposition 2.1 we only need to exhibit two elements $v, w \in E\left(A_{n}^{*}\right)$, such that $v \approx_{n, m, k} w$ and $v \nsim_{n, m, k} w$.

Let $v$ and $w$ be, respectively, the paths

$$
\begin{aligned}
& \left(x u_{1}\right)^{k_{1}} y_{1} u_{2}\left(z_{1} u_{1} y_{1} u_{2}\right)^{k_{2}} y_{2} u_{3}\left(z_{2} u_{2} y_{2} u_{3}\right)^{k_{3}} y_{3} u_{4} \cdots\left(z_{n} u_{n} y_{n} u_{n+1}\right)^{k_{n+1}} t\left(u_{n+1} t\right)^{k_{n+2}}, \\
& \left(x u_{1}\right)^{l_{1}} y_{1} u_{2}\left(z_{1} u_{1} y_{1} u_{2}\right)^{l_{2}} y_{2} u_{3}\left(z_{2} u_{2} y_{2} u_{3}\right)^{l_{3}} y_{3} u_{4} \cdots\left(z_{n} u_{n} y_{n} u_{n+1}\right)^{l_{n+1}} t\left(u_{n+1} t\right)^{l_{n+2}},
\end{aligned}
$$

where, for even $i, k_{i}=m+k$ and $l_{i}=m$, and, for odd $i, k_{i}=m$ and $l_{i}=m+k$. In these expressions, the first appearance of $u_{i}$ is in fact $u_{i}^{1}$, the second $u_{i}^{2}$, and so on. By definition, $v \approx_{n, m, k} w$ and $v \nsim_{n, m, k} w$.

Denote by $\eta_{n}$ the identity over the graph $A_{n}$ whose sides are the paths (5.1) and (5.2) in the proof of Proposition 5.6. We have in fact verified the following result.

Corollary 5.7. The identity $\eta_{n}$ is valid in the pseudovariety $g \mathbf{C o m}_{m, k}$ but not in the category $C_{n, m, k}$.

\subsection{A minimality property of $C_{n, m, k}$ for $m \geqslant 2$}

We can now establish an analogue for $C_{n, m, k}$ of Proposition 3.3. Although this result will not be used elsewhere in the paper, it is included for the sake of motivation. Indeed, it was this property of $C_{n, m, k}$ that led to its discovery and the fact that $g \mathbf{C o m}_{m, k}$ has infinite vertex rank for an integer $m$ greater than 1 and $k \in \mathbb{P}$ was deduced from it in preliminary versions of this paper. Now, of course, we have the much stronger Theorem 3.4.

Proposition 5.8. Let $n, k \geqslant 1$ and $m \geqslant 2$, and let $D$ be a subcategory of $C_{n, m, k}$ such that $V(D) \neq V\left(C_{n, m, k}\right)$. Then $D \in g \mathbf{C o m}_{m, k}$.

Proof. Suppose that for some $r \in\{2,3, \ldots, n\}, p_{r} \notin V(D)$ (the remaining cases can be treated similarly). We may assume that $D$ is the largest subcategory of $C_{n, m, k}$ such that $V(D)=V\left(C_{n, m, k}\right) \backslash\left\{p_{r}\right\}$.

Let $B_{n}$ be the graph

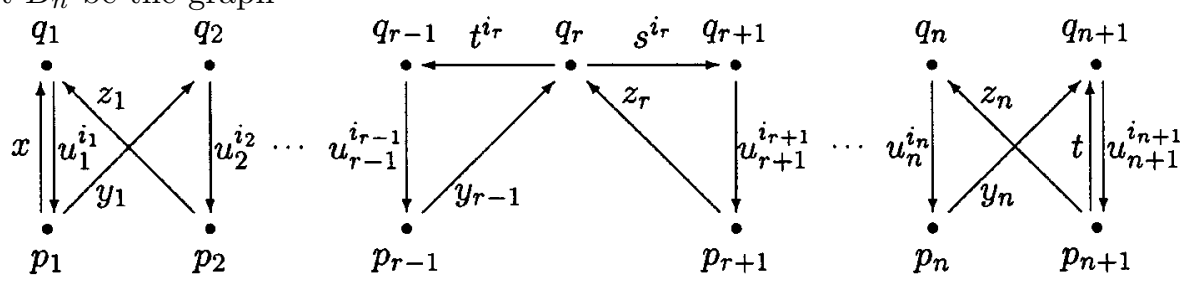


where, for each lower index $j, u_{j}^{i_{j}}$ again indicates one of several coterminal edges with the upper index running over the corresponding set as follows: $i_{1}, i_{n+1} \in\{1,2, \ldots, 2 m+k\}$ and, for $j \notin\{1, r, n+1\}, i_{j} \in\{1,2, \ldots, 2 m+k+1\}$. Consider the congruence (of finite index) $\simeq_{n}$ on the free category $B_{n}^{*}$ defined as follows. For coterminal edges $u$ and $v$ of $B_{n}^{*}, v \simeq_{n} w$ if at least one of the following conditions holds:

$$
\begin{gathered}
(\exists i, l)|v|_{u_{i}^{l}} \geqslant 2 \text { and }(\exists i, l)|w|_{u_{i}^{l}} \geqslant 2, \\
(\exists i)|v|_{t^{i}}+|v|_{s^{i}} \geqslant 2 \text { and }(\exists i)|w|_{t^{i}}+|w|_{s^{i}} \geqslant 2, \\
\left(\forall a \in E\left(B_{n}\right)\right)|v|_{a}=|w|_{a} .
\end{gathered}
$$

Let $E_{n}=B_{n}^{*} / \simeq_{n}$.

Consider the following diagram of functors:

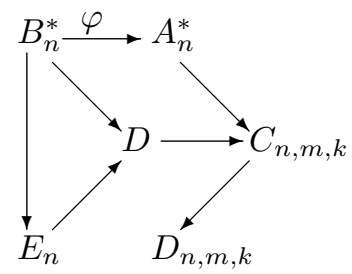

The functor $\varphi$ is the inclusion mapping on the common part of $B_{n}$ and $A_{n}$ and sends the edges $t^{i_{r}}$ and $s^{i_{r}}$, respectively, to $u_{r}^{i_{r}} z_{r-1}$ and $u_{r}^{i_{r}} y_{r}$. The functors $B_{n}^{*} \rightarrow E_{n}, A_{n}^{*} \rightarrow C_{n, m, k}$ and $C_{n, m, k} \rightarrow D_{n, m, k}$ are the natural quotient functors. The functor $D \rightarrow C_{n, m, k}$ is the inclusion functor. The existence of a quotient functor $B_{n}^{*} \rightarrow D$ such that the diagram commutes follows from the hypothesis that $D$ is the largest subcategory of $C_{n, m, k}$ which misses the vertex $p_{r}$. The existence of a quotient functor $E_{n} \rightarrow D$ such that the diagram commutes follows from the definition of $\simeq_{n}$. In particular, to show that $D \in g \mathbf{C o m}_{m, k}$, it suffices to prove that $E_{n} \in g \mathbf{C o m}_{m, k}$.

By Proposition 2.1, to complete the proof it suffices to show that $\equiv_{m, k} \subseteq \simeq_{n}$. Let $v, w \in B_{n}^{*}$ be coterminal edges such that $v \equiv_{m, k} w$ and suppose that both (5.3) and (5.4) fail. We must show that (5.5) holds. Indeed, then $\varphi(v) \equiv_{m, k} \varphi(w)$ and, since $m \geqslant 2$, $|v|_{s^{l}} \geqslant 2$ if and only if $|w|_{s^{l}} \geqslant 2$, which implies that $|v|_{s^{l}}=|w|_{s^{l}} \leqslant 1$ for all $l$. This yields

$$
|\varphi(v)|_{y_{r}}=\sum_{l}|v|_{s^{l}}=\sum_{l}|w|_{s^{l}}=|\varphi(w)|_{y_{r}} .
$$

By Proposition 5.4, $\varphi(v) \sim_{n, m, k} \varphi(w)$ and so $v \simeq_{n} w$.

Although the proof is already complete, to clarify the situation we complete the above diagram with an injective functor $E_{n} \rightarrow D_{n, m, k}$ such that the diagram commutes. Indeed, if $v, w \in B_{n}^{*}$ are coterminal edges such that $\varphi(v)$ and $\varphi(w)$ are non-zero, then the argument in the preceding paragraph shows that $\varphi(v) \equiv_{m, k} \varphi(w)$ if and only if $v \simeq_{n} w$.

\subsection{Some further properties of $C_{n, m, k}$}

In this subsection we gather a few more properties of the categories $C_{n, m, k}$ which will be used in the next subsection. Specifically we show that $C_{n, m, k}$ satisfies certain identities. 
Lemma 5.9. Let $v$ and $w$ be two coterminal paths of the graph $A_{n}$ with the same content such that $v$ and $w$ represent two distinct non-zero edges of $C_{n, m, k}$. Then

$$
|v|_{y_{i}} \neq|w|_{y_{i}} \quad \text { and } \quad|v|_{z_{i}} \neq|w|_{z_{i}} \quad \text { for every } i \in\{0,1, \ldots, n+1\} .
$$

Proof. By Proposition 5.4, there must be some $s \in\{0,1, \ldots, n+1\}$ such that $|v|_{y_{i}} \neq$ $|w|_{y_{i}}$. By Corollary 5.3, we have in fact (5.6).

Proposition 5.10. For $m \geqslant 2$ and $r \neq n$, the category $C_{n, m, k}$ satisfies the identity $\eta_{r}$.

Proof. Evaluate the graph $A_{r}$ in $C_{n, m, k}$ through a graph homomorphism, thus obtaining for the sides of the identity $\eta_{r}$ two edges represented by coterminal paths $v$ and $w$ of $A_{n}^{*}$. Suppose that $v \chi_{n, m, k} w$. Since $g \mathbf{C o m}_{m, k}$ satisfies $\eta_{r}$ by Corollary 5.7, we know that $v \approx_{n, m, k} w$ and, therefore, $v$ and $w$ represent non-zero edges of $C_{n, m, k}$. By Lemma 5.9, we deduce that (5.6) holds.

To finish the proof, it suffices to show that (5.6) together with $v \chi_{n, m, k} w$ is impossible for $r \neq n$. Indeed, the edges $y_{i}$ and $z_{i}(i \in\{0,1, \ldots, n+1\})$ of the graph $A_{r}$ must evaluate to the same type of edges in $A_{n}$, because otherwise both sides of $\eta_{r}$ would evaluate to the same zero edge. If some $u_{i}^{l} \in E\left(A_{r}\right)$ did evaluate to a path involving an edge $y_{j}$ or an edge $z_{j}$, then, since $v$ and $w$ are non-zero edges, we would have $|v|_{y_{j}}=1=|w|_{z_{j}}$, in contradiction with (5.6). Hence all edges of $A_{r}$ must be mapped to the same type of edges of $A_{n}$, and so the evaluation is actually defined by a graph homomorphism $A_{r} \rightarrow A_{n}$. Since it is easily verified that there is no such graph homomorphism for $r \neq n$, we reach a contradiction. Hence $C_{n, m, k}$ satisfies $\eta_{r}$.

Proposition 5.11. For an integer $m \geqslant 2$, the category $C_{n, m, k}$ lies in any pseudovariety of the form $g \mathbf{C o m}_{r, \ell}$, which strictly contains $g \mathbf{C o m}_{m, k}$.

Proof. Note that the hypothesis that $g \mathbf{C o m}_{r, \ell} \supsetneqq g \mathbf{C o m}_{m, k}$ means that $m \leqslant r$, $k$ divides $\ell$, and at least one of these relations is strict.

Suppose that $v$ and $w$ are two coterminal paths of $A_{n}$ such that $v \equiv_{r, \ell} w$. We must show that $v \sim_{n, m, k} w$. Suppose that, on the contrary, $v \chi_{n, m, k} w$ so that, in particular, $v$ and $w$ are non-zero edges. Then, by Lemma 5.9, condition (5.6) holds and so, since $v \equiv_{r, \ell} w$, all $|v|_{y_{i}},|w|_{y_{i}},|v|_{z_{i}}$ and $|w|_{z_{i}}$ are at least $r$ and some of them must be at least $r+\ell$, and in particular strictly greater than $m+k$. By Lemma 5.2, we also know that, for $i \in\{1, \ldots, n+1\}$,

$$
|v|_{i}=|v|_{y_{i}}+|v|_{z_{i-1}}+\xi_{i} \text { and }|w|_{i}=|w|_{y_{i}}+|w|_{z_{i-1}}+\xi_{i},
$$

where $\xi_{i} \in\{-1,0,1\}$ are given by Lemma 5.2. Moreover, since $v$ and $w$ are non-zero edges in $C_{n, m, k}$, we must have $|v|_{i},|w|_{i} \leqslant 2 m+k+1$. Then, if say $|v|_{y_{i}}>|w|_{y_{i}}$, we conclude from

$$
2 m+k+1 \geqslant|v|_{i}=|v|_{y_{i}}+|v|_{z_{i-1}}+\xi_{i}>2 m+k+1+\xi_{i}
$$

that $\xi_{i}=-1$ and $|v|_{z_{i-1}}=m$. Hence, in view of Lemma 5.2, $\alpha(v)=\alpha(w)=p_{i}$. The same conclusion is obtained analogously under the assumption $|v|_{y_{i}}<|w|_{y_{i}}$. This shows that 
there is at most one $i \in\{1, \ldots, n+1\}$ such that $|v|_{y_{i}} \neq|w|_{y_{i}}$, which is a contradiction since we already observed that this relation holds for every $i$ and $n \geqslant 1$. Hence $v \sim_{n, m, k} w$.

\subsection{Big gaps}

For a pseudovariety of semigroupoids $\boldsymbol{V}$, say that a family $\mathcal{F}$ of semigroupoids is independent modulo $\boldsymbol{V}$ if no $S \in \mathcal{F}$ belongs to the pseudovariety generated by $\boldsymbol{V} \cup(\mathcal{F} \backslash$ $\{S\})$.

Theorem 5.12. For all $m \geqslant 2$ and $k \geqslant 1$, the family of semigroupoids $\left\{C_{n, m, k}: n \geqslant\right.$ $1\}$ is independent modulo $g \mathbf{C o m}_{m, k}$.

Proof. This follows immediately from Corollary 5.7 and Proposition 5.10.

Another independent family may be extracted from the categories $L_{r, m}$ of $\S 3$. Denote by $K_{r, m}$ the semigroupoid obtained from $L_{r, m}$ by removing all local identities.

Theorem 5.13. For every $m \geqslant 2$, the family of semigroupoids $\left\{K_{r, m}: r \geqslant 2\right\}$ is independent modulo $g \mathbf{C o m}_{m, \omega}$.

Proof. In view of the fact, established in the proof of Proposition 3.2, that $K_{r, m}$ fails the pseudoidentity $\varepsilon_{r, m, \omega}$, which in turn is valid in $g \mathbf{C o m}_{m, \omega}$, it suffices to observe that $K_{r, m}$ satisfies $\varepsilon_{s, m, 1}$ (and therefore also $\varepsilon_{s, m, \omega}$ ) for $s \neq r$.

Let $\varphi: G_{s} \rightarrow K_{r, m}$ be a graph homomorphism evaluating the underlying graph of the pseudoidentity $\varepsilon_{s, m, 1}$ in $K_{r, m}$. If $s<r$, then $\varphi$ assumes its values in a subsemigroupoid $C$ of $K_{r, m}$ with at most $s$ vertices, and so $C \in g \mathbf{C o m}_{m, 1}$ by Proposition 3.3. Hence $C$ satisfies $\varepsilon_{s, m, 1}$, since this pseudoidentity is valid in $g \mathbf{C o m}_{m, 1}$ and, therefore, $K_{r, m}$ satisfies $\varepsilon_{s, m, 1}$. Suppose next that $s>r$. If any edge $x_{i}$ of the graph $G_{s}$ is mapped under $\varphi$ to an edge of $K_{r, m}$ involving some $y_{j}$, then clearly both sides of $\varepsilon_{s, m, 1}$ evaluate to a zero in $K_{r, m}$. Otherwise, two of the edges $x_{i}$ of the graph $G_{s}$ must be mapped to edges of $K_{r, m}$ containing the same $x_{\ell}$ in their content. Taking into account the defining relations of the category $L_{r, m}$, this again implies that both sides of $\varepsilon_{s, m, 1}$ must be evaluated to a zero in $K_{r, m}$. Hence $K_{r, m}$ satisfies $\varepsilon_{s, m, 1}$.

Say that an interval $[\boldsymbol{V}, \boldsymbol{W}]$ of pseudovarieties is a big gap if it contains a chain isomorphic to the chain of real numbers under the usual order as well as a continuum anti-chain. A standard argument which may be found in [7, Proposition 1.1] for pseudovarieties of semigroups gives the following result.

Proposition 5.14. Let $\boldsymbol{V}$ and $\boldsymbol{W}$ be two pseudovarieties of semigroupoids such that $\boldsymbol{V} \subseteq \boldsymbol{W}$ and $\boldsymbol{W}$ contains an infinite family which is independent modulo $\boldsymbol{V}$. Then the interval $[\boldsymbol{V}, \boldsymbol{W}]$ is a big gap.

We may finally establish the main results of this section.

Theorem 5.15. For all $m \geqslant 2$ and $k \geqslant 1$, the interval of categorical pseudovarieties between $g \mathbf{C o m}_{m, k}$ and any of its successor skeleton points (namely $g \mathbf{C o m}_{m+1, k}$ and $g \mathbf{C o m}_{m, k p}$ for a prime $p$ ) is a big gap. 
Proof. This follows from Proposition 5.11 and Theorem 5.12, in view of Proposition 5.14.

We do not know whether Theorem 5.15 remains valid for $m=0$ or $m=1$. An extension of part of Theorem 5.15 is obtained by considering our other independent family. But since semigroupoids are used instead of categories, we can no longer guarantee that the pseudovarieties in the chain can be chosen to be categorical.

Theorem 5.16. For all $m \geqslant 2$ and $\pi \in \hat{\mathbb{P}}$, the interval $\left[g \mathbf{C o m}_{m, \pi}, g \mathbf{C o m}_{m+1, \pi}\right]$ is a big gap.

Proof. Since the family $\left\{K_{r, m}: r \geqslant 2\right\}$ is independent modulo $g \mathbf{C o m}_{m, \omega}$ by Theorem 5.13, it is also independent modulo the smaller pseudovariety $g \mathbf{C o m}_{m, \pi}$. On the other hand, since all $K_{r, m}$ lie in $g \mathbf{C o m}_{m+1,1}$ by Proposition 3.2, they also belong to the larger pseudovariety $g \mathbf{C o m}_{m+1, \pi}$. Hence the result follows from Proposition 5.14.

\section{The semigroup case}

As observed earlier, the pseudovarieties $\mathbf{C o m}_{m, \pi}(m \in \mathbb{N} \cup\{\omega\}, \pi \in \hat{\mathbb{P}})$ are precisely the monoidal pseudovarieties of commutative semigroups and constitute a (complete) sublattice of the lattice $\mathcal{P} s(\mathbf{C o m})$. In general, a pseudovariety $\boldsymbol{V}$ of commutative semigroups is the join of the largest pseudovariety in this sublattice contained in $\boldsymbol{V}$ (which we now call the monoidal part of $\boldsymbol{V}$ ) with $\boldsymbol{V} \cap \boldsymbol{N}$, where $\boldsymbol{N}$ denotes the pseudovariety of all finite nilpotent semigroups (cf. $[\mathbf{3}, \S 6.2])$.

In this section, we discuss the extension of the results of $\S \S 3$ and 4 from monoidal pseudovarieties of commutative semigroups to the case of arbitrary pseudovarieties $\boldsymbol{V}$ of commutative semigroups. For the finite basis problem, in view of Theorem 3.4, the problem is only of interest if the Nelson index of $\boldsymbol{V}$ is 0,1 or $\omega$. Moreover, in the latter case, the monoidal part of $\boldsymbol{V}$ contains $\boldsymbol{N}$ and so $\boldsymbol{V}$ is monoidal and, therefore, $g \boldsymbol{V}$ is finitely based by Theorem 3.5. The cases where the Nelson index is 0 or 1 correspond, respectively, to

$$
\boldsymbol{V} \subseteq \llbracket x^{\omega}=y^{\omega}, x y=y x \rrbracket
$$

and

$$
\boldsymbol{S l} \subseteq \boldsymbol{V} \subseteq \llbracket x^{\omega} y^{\omega+1}=x^{\omega} y, x y=y x \rrbracket,
$$

where $\boldsymbol{S} \boldsymbol{l}=\mathbf{C o m}_{1,1}$ denotes the pseudovariety of all finite semilattices (cf. $[\mathbf{3}, \S 9.1]$ ). While we have not treated all these cases systematically, we present some examples to illustrate the difficulties in a systematic treatment of the problem.

As to decidability of $g \boldsymbol{V}$ with $\boldsymbol{V} \subseteq \mathbf{C o m}$, a lot more cases remain to be treated. We illustrate the problem with specific examples.

The first example gives another application of the combinatorial Lemma 4.2.

Proposition 6.1. $g(\boldsymbol{N} \cap \mathrm{Com})=g \operatorname{Com} \cap g N=g \operatorname{Com} \cap \ell N$. 
Proof. Since the operator $g$ preserves order, the inclusions from left to right are immediate. For the closing wrapped inclusion, suppose that $g(\boldsymbol{N} \cap \mathbf{C o m})$ satisfies a semigroupoid pseudoidentity $(u=v ; \Gamma)$. Then either $|u|_{a}=|v|_{a} \in \mathbb{N}$ for all $a \in E(\Gamma)$, and so the pseudoidentity is valid in $g$ Com, or $|u|_{a},|v|_{b} \in \hat{\mathbb{P}} \backslash \mathbb{P}$ for some $a, b \in E(\Gamma)$. Then, by Lemma 4.2 it follows that $g \mathbf{C o m} \cap \ell \boldsymbol{N}$ satisfies $(u=v ; \Gamma)$.

The second example is another case of a nilpotent pseudovariety with unbounded nilpotent index but small nil index.

To avoid writing too many pseudoidentities, we introduce some abbreviations. Besides the already defined pseudoidentities, we will consider shorthand pseudoidentities of the form $(u=\overline{0} ; \Gamma)$. Whenever the shorthand pseudoidentities $(u=\overline{0} ; \Gamma)$ and $(v=\overline{0} ; \Delta)$ are found in a set of pseudoidentities, the real pseudoidentity $(u=v ; \Upsilon)$ should be read where $\Upsilon$ is the graph that is obtained from the disjoint union $\Gamma \dot{\cup} \Delta$ by identifying $\alpha u$ with $\alpha v$ and $\omega u$ with $\omega v$. In this convention, we do not exclude the possibility that $(u=\overline{0} ; \Gamma)=(v=\overline{0} ; \Delta)$.

Proposition 6.2. Let $\boldsymbol{V}=\llbracket x^{\omega}=0, x^{2} y=x y^{2}, x y=y x \rrbracket$. Then

$$
\begin{aligned}
& g \boldsymbol{V}=g \operatorname{Com} \cap \llbracket\left(x^{2} y=x y^{2} ; \bullet x, y\right),\left(x^{2} y z=\overline{0} ; x \text { C } \stackrel{y}{\longrightarrow} \stackrel{z}{\longrightarrow} \cdot\right),
\end{aligned}
$$

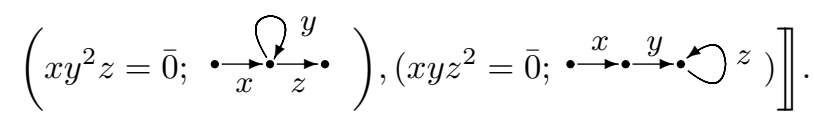

Proof. Note that $\boldsymbol{V}$ satisfies the following pseudoidentities

$$
x^{2} y^{2}=x y^{4}=x y^{2} y^{2}=x^{2} y y^{2}=x^{2} y^{2} y=x^{2} y^{2} y^{2}=\cdots=x^{2} y^{2} y^{\omega}=0
$$

and so also

$$
x^{2} y z=x(y z)^{2}=x y^{2} z^{2}=0 .
$$

It follows that a non-trivial pseudoidentity $u=v$ is valid in $\boldsymbol{V}$ if and only if for each side there is some variable occurring more than once and either the length of each side is at least 4 or both sides have length 3 and the same content. The result follows easily.

The last two examples contain all finite abelian groups.

Proposition 6.3. The global $g \llbracket x^{\omega}=y^{\omega}, x y=y x \rrbracket$ is given by

$$
g \operatorname{Com} \cap \llbracket\left(x^{\omega} y=y z^{\omega} ;{ }^{x} C \cdot \stackrel{y}{\longrightarrow} \bigcirc z\right) \rrbracket .
$$

Proof. The result may be deduced from the observation that a pseudoidentity $u=v$ is valid in $\llbracket x^{\omega}=y^{\omega}, x y=y x \rrbracket$ if and only if, for every variable $a$ such that $|u|_{a} \neq|v|_{a}$, there are variables $b$ and $c$ such that $|u|_{b},|v|_{c} \notin \mathbb{N}$ and there is some $k \in \mathbb{N}$ such that $\left\{|u|_{a},|v|_{a}\right\}=\{k, \omega+k\}$. The details are omitted.

Proposition 6.4. The global $g \llbracket x^{\omega} y^{\omega+1}=x^{\omega} y, x y=y x \rrbracket$ is given by

$$
g \operatorname{Com} \cap \llbracket\left(x^{\omega+1} y z=x y z^{\omega+1} ; x \subset \cdot \stackrel{y}{\rightarrow} \bigcirc z\right) \rrbracket .
$$


Proof. Here the result follows from the observation that a pseudoidentity $u=v$ is valid in $\llbracket x^{\omega} y^{\omega+1}=x^{\omega} y, x y=y x \rrbracket$ if and only if $c(u)=c(v)$ and, for every variable $a$ such that $|u|_{a} \neq|v|_{a}$, there are variables $b$ and $c$ such that $|u|_{b},|v|_{c} \notin \mathbb{N}$ and there is some $k \in \mathbb{N}$ such that $\left\{|u|_{a},|v|_{a}\right\}=\{k, \omega+k\}$. The details are omitted.

The above examples suggest the guess that globals of pseudovarieties contained in $\llbracket x^{\omega} y^{\omega+1}=x^{\omega} y, x y=y x \rrbracket$ are all finitely based and are decidable if and only if their group part is decidable. A complete proof of such a result along the ad hoc lines hinted at in the examples is perhaps too tedious, even if it is feasible.

It should also be observed that the global operator $g$ does not preserve intersections. For example, for $m \geqslant 2$ and distinct primes $p$ and $q$, by Theorem 4.1 the intersection $g \mathbf{C o m}_{m, p^{\omega}} \cap g \mathbf{C o m}_{m, q^{\omega}}$ is defined by the pseudoidentity (2.1) defining $g$ Com along with the pseudoidentities $\varepsilon_{r, m, p^{\omega}}$ and $\varepsilon_{r, m, q^{\omega}}$ with $r \geqslant 2$, which together imply $\varepsilon_{r, m, 1}$. Since there are no circuits in the categories $C_{n, m, k}$ of $\S 5.1$ that use none of the edges $u_{i}^{j}$, it is easy to see that $C_{n, m, k}$ satisfies the pseudoidentities $\varepsilon_{r, m, 1}$. Hence

$$
g \mathbf{C o m}_{m, p^{\omega}} \cap g \operatorname{Com}_{m, q^{\omega}} \neq g \mathbf{C o m}_{m, 1}=g\left(\mathbf{C o m}_{m, p^{\omega}} \cap \mathbf{C o m}_{m, q^{\omega}}\right) .
$$

In contrast, as observed in [10], if the five-element aperiodic Brandt semigroup $B_{2}$ belongs to the pseudovarieties $\boldsymbol{V}_{i}(i \in I)$, then

$$
g \bigcap_{i} \boldsymbol{V}_{i}=\bigcap_{i} g \boldsymbol{V}_{i}
$$

On the other hand, the operator $g$ preserves joins, as can be very easily checked from the definitions. Yet the computation of joins is in general quite hard, and so it is perhaps not reasonable to try to compute $g \boldsymbol{V}$ by computing $g(\boldsymbol{V} \cap \boldsymbol{N})$ and taking the join with the global of the monoidal part.

Acknowledgements. This work was supported, in part, by the project Praxis/2/ s2.1/MAT/63/94. Support for J.A. from Fundação para a Ciência e a Tecnologia through the Centro de Matemática, University of Porto, Porto, Portugal, is gratefully acknowledged as is support for A.A. from FCT through the Centro de Matemática, University of Minho, Braga, Portugal.

\section{References}

1. J. Almeida, Some order properties of the lattice of varieties of commutative semigroups, Can. J. Math. 38 (1986), 19-47.

2. J. Almeida, Semidirect products of pseudovarieties from the universal algebraist's point of view, J. Pure Appl. Algebra 60 (1989), 113-128.

3. J. Almeida, Finite semigroups and universal algebra (World Scientific, Singapore, 1995). (English translation.)

4. J. Almeida, Hyperdecidable pseudovarieties and the calculation of semidirect products, Int. J. Algebra Comput. 9 (1999), 241-261.

5. J. Almeida And B. Steinberg, On the decidability of iterated semidirect products and applications to complexity, Proc. Lond. Math. Soc. 80 (2000), 50-74. 
6. J. Almeida And B. Steinberg, Syntactic and global semigroup theory, a synthesis approach, in Algorithmic Problems in Groups and Semigroups (ed. J. C. Birget, S. W. Margolis, J. Meakin and M. V. Sapir), pp. 1-23 (Birkhäuser, Basel, 2000).

7. J. Almeida And M. V. Volkov, The gap between partial and full, Int. J. Algebra Comput. 8 (1998), 399-430.

8. J. Almeida And P. Weil, Relatively free profinite monoids: an introduction and examples, in Semigroups, formal languages and groups (ed. J. B. Fountain), vol. 466, pp. 73-117 (Dordrecht, Kluwer, 1995).

9. J. Almeida And P. Weil, Profinite categories and semidirect products, J. Pure Appl. Algebra 123 (1998), 1-50.

10. J. Almeida, A. Azevedo and L. Teixeira, On finitely based pseudovarieties of the forms $\boldsymbol{V} * \boldsymbol{D}$ and $\boldsymbol{V} * \boldsymbol{D}_{n}$, J. Pure Appl. Algebra 146 (2000), 1-15.

11. J. A. Brzozowski And I. Simon, Characterizations of locally testable events, Discrete Math. 4 (1973), 243-271.

12. P. R. Jones and P. G. Trotter, Locality of DS and associated varieties, J. Pure Appl. Algebra 104 (1995), 275-301.

13. R. Knast, Some theorems on graph congruences, RAIRO Inf. Théor. Appl. 17 (1983), $331-342$.

14. E. NeLSON, The lattice of equational classes of commutative semigroups, Can. J. Math. 33 (1971), 875-895.

15. H. Straubing, Finite semigroup varieties of the form $V * D$, J. Pure Appl. Algebra 36 (1985), 53-94.

16. D. ThÉRIEn And A. Weiss, Graph congruences and wreath products, J. Pure Appl. Algebra 36 (1985), 205-215.

17. B. Tilson, Categories as algebra: an essential ingredient in the theory of monoids, $J$. Pure Appl. Algebra 48 (1987), 83-198. 